\title{
Learning to Fold RNAs in Linear Time
}

\author{
F A Rezaur Rahman Chowdhury ${ }^{1,3},{ }^{\star}$, He Zhang ${ }^{1,{ }^{\star}}$, and Liang Huang ${ }^{1,2}$ \\ 1 Baidu Research USA, Sunnyvale, CA 94089, USA, \\ ${ }^{2}$ School of Electrical Engineering \& Computer Science, Oregon State University, Corvallis, OR 97330, USA, \\ 3 Washington State University
}

\begin{abstract}
RNA secondary structure is helpful for understanding RNA's functionality, thus accurate prediction systems are desired. Both thermodynamics-based models and machine learning-based models have been used in different prediction systems to solve this problem. Compared to thermodynamicsbased models, machine learning-based models can address the inaccurate measurement of thermodynamic parameters due to experimental limitation. However, the existing methods for training machine learning-based models are still expensive because of their cubic-time inference cost. To overcome this, we present a linear-time machine learning-based folding system, using recently proposed approximate folding tool LinearFold as inference engine, and structured SVM (sSVM) as training algorithm. Furthermore, to remedy non-convergence of naive SSVM with inexact search inference, we introduce a max violation update strategy. The training speed of our system is $41 \times$ faster than CONTRAfold on a diverse dataset for one epoch, and $14 \times$ faster than MXfold on a dataset with longer sequences. With the learned parameters, our system improves the accuracy of LinearFold, and is also the most accurate system among selected folding tools, including CONTRAfold, Vienna RNAfold and MXfold.
\end{abstract}

Keywords: RNA folding, machine learning, structured SVM, max violation, linear time

\section{Introduction}

For past decades, our understanding of ribonucleic acid (RNA) is changing. Proofs reveal that RNAs are involved in multiple processes, including gene expression, RNA modifications guiding [8] and particular diseases regulating [13]. RNA's functionalities are highly related to its secondary structures, but determining the secondary structure using experimental methods is expensive, time-comsuming and difficult. Therefore, being able to rapidly and accurately predict RNA secondary structures is very useful and desired.

Accurate prediction model, i.e., well-designed features (for example base pair CG and AU, terminal mismatches, etc) and their weights, is one of the keys for solving RNA secondary structure prediction problem. Both thermodynamics-based models and machine learning-based models have been proposed and used for this problem, they share similar features, but use different ways to get feature weights. Thermodynamicsbased models get feature weights directly from experimentally estimated thermodynamic parameters, and these models are widely used by RNA secondary structure prediction engines, such as Vienna RNAfold [15] and RNAstructure [17]. Thermodynamics-based models and engines constitute the most popular way for secondary structure prediction, however, they may suffer from the inaccurate measurement of thermodynamic parameters due to experimental limitation, which leads to an inaccurate prediction result [6].

Alternatively, machine learning-based models borrow feature templates from thermodynamics-based models, but use machine learning techniques to learn feature weights from known structures. The first trials are to borrow stochastic context-free grammars (SCFGs) learning framework 7]11/12 from natural languaging processing field, but due to the weak feature expression ability of SCFGs, their accuracies are all lower than thermodynamics-based models. To further improve accuracy, CONTRAfold $\underline{6}$ proposes a new machine learning-based method using Conditional Random Field (CRF) [14 for training, and achieves higher accuracy than thermodynamic methods. However, CONTRAfold training is very slow due to CRF algorithm, which makes it impossible for training on big dataset. A more recent machine learning-based work, MXfold [1], uses structured SVM [19] instead of CRF to accelarate the training process. Though it is faster than CONTRAfold training, it borrows the inference implementation from CONTRAfold, which runs in cubic time and results in a costly inference process, especially when the training set includes long sequences. In

\footnotetext{
* equal contribution
} 
Rezaur Chowdhury et al.

\begin{tabular}{c|c|c|c}
\hline & \multicolumn{3}{|c}{ Training } \\
\hline System & Method & Inference Algorithm & Speed \\
\hline CONTRAfold & Conditional Random Field & $O\left(n^{3}\right)$ & slowest \\
MXfold & structured SVM & $O\left(n^{3}\right)$ & medium \\
Our work & structured SVM & LinearFold & fastest \\
\hline
\end{tabular}

Table 1: Comparison of three machine learning-based RNA folding systems.

addition, MXfold is not a pure machine learning-based model since it integrates thermodynamic model with learned model.

To overcome the efficiency bottleneck of training, we present a new machine learning-based RNA folding system, using the recently proposed linear-time prediction engine LinearFold [10] for inference. We use structured SVM algorithm for training. However, using LinearFold as inference may result in invalid updates due to the inexact search nature of LinearFold, and break the convergence property in naive sSVM training process. To remedy this, we utilize a max violation update strategy originally from violation-fixing structured perceptron [3/4, and generalize the naive sSVM algorithm to an advanced version of sSVM.

The results show that the training speed of our system is $41 \times$ faster than CONTRAfold for one epoch on a diverse dataset (average length $208.6 \mathrm{nt}$ ). On a dataset with more long sequences, our system is $14 \times$ faster than MXfold (average length 2713.5 nt). With the learned parameters, our system is the most accurate system among selected folding tools, including CONTRAfold, Vienna RNAfold and MXfold. Using a cross validation training, Our system improves the off-the-shelf LinearFold-C by $+4.62 \%$ in PPV and $+8.55 \%$ in sensitivity. Compared with retrained MXfold, our system gains $+3.51 \%$ and $+4.70 \%$ on PPV and sensitivity, separately.

Table 1 summarize the differences between 3 systems.

Our contributions are as followed:

- We propose a fast training system which can do training and inference both in linear time. Our system can make big dataset training and long sequences training much more efficient.

- With the learned parameters, our system is more accurate compared with existing systems.

- We prove that sSVM converges when doing exact search for inference, but the convergence property does not hold when the search is inexact. We use a max violation update strategy to solve the convergence problem.

\section{Methods}

\subsection{Structured SVM Training with Linear-Time Inference}

We use structured SVM (sSVM) for training and LinearFold for linear-time inference. This makes the training process faster than both CONTRAfold and MXfold, especially when the training dataset contains long sequences.

Formally, we define $\mathcal{D}$ as the training dataset, and $(x, y)$ as RNA sequence and its structure in $\mathcal{D}$. $\mathcal{Y}(x)$ is the set of all possible structures for the given sequence $x . y^{\prime}$ is a predicted structure, i.e., $y^{\prime} \in \mathcal{Y}(x)$. sSVM training is to learn a feature weight vector $\mathbf{w}$, which scores the native structure $y$ higher than $y^{\prime}$ by a margin of $\Delta\left(y, y^{\prime}\right)$ :

$$
\forall(x, y) \in \mathcal{D}, \forall y^{\prime} \in \mathcal{Y}(x), \mathbf{w} \cdot \mathbf{\Phi}(x, y)-\mathbf{w} \cdot \mathbf{\Phi}\left(x, y^{\prime}\right) \geq \Delta\left(y, y^{\prime}\right)
$$

where $\boldsymbol{\Phi}$ is the feature function, mapping $(x, y)$ (or $\left.\left(x, y^{\prime}\right)\right)$ to its feature vector. $\Delta\left(y, y^{\prime}\right)$ is the decomposable augmented loss to measure the difference between $y$ and $y^{\prime}$. We define $\Delta\left(y, y^{\prime}\right)$ as:

$$
\Delta\left(y, y^{\prime}\right)=\alpha \mathrm{FP}\left(y, y^{\prime}\right)+\beta \mathrm{FN}\left(y, y^{\prime}\right)
$$




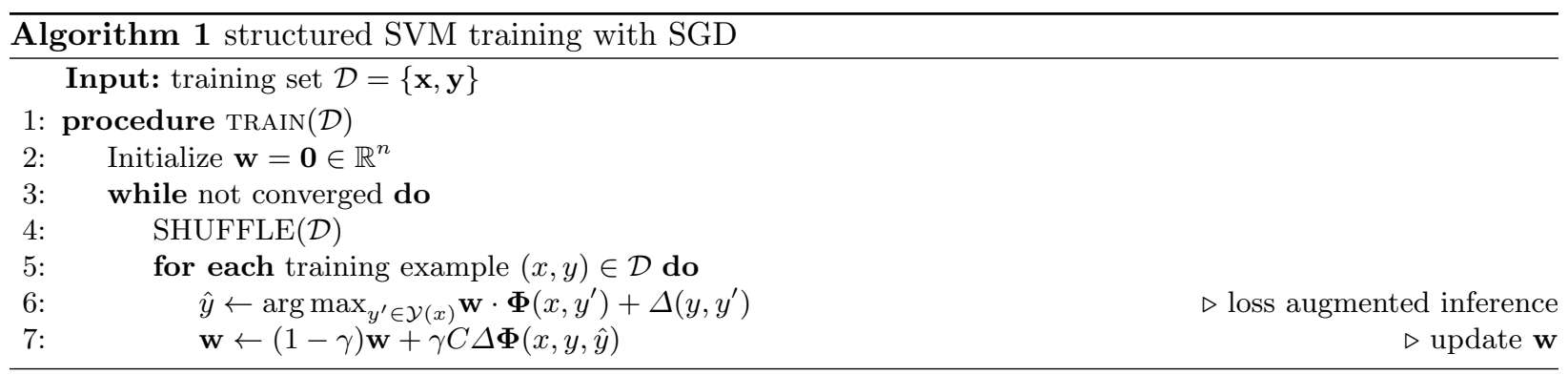

where $\mathrm{FP}\left(y, y^{\prime}\right)$ is the number of False Positive pairs and $\mathrm{FN}\left(y, y^{\prime}\right)$ is the number of False Negative pairs. $\alpha$ and $\beta$ are hyper-parameters that balance PPV and sensitivity.

For simplicity, we define $\Delta \boldsymbol{\Phi}\left(x, y, y^{\prime}\right)$ as the feature vector difference of $y$ and $y^{\prime}$, i.e., $\Delta \boldsymbol{\Phi}\left(x, y, y^{\prime}\right):=$ $\mathbf{\Phi}(x, y)-\mathbf{\Phi}\left(x, y^{\prime}\right)$. A triple $\left(x, y, y^{\prime}\right)$ is called to be a violation with respect to $\mathbf{w}$ if:

$$
\mathbf{w} \cdot \Delta \boldsymbol{\Phi}\left(x, y, y^{\prime}\right)<\Delta\left(y, y^{\prime}\right)
$$

Among all the violations, we define the most violated structure $\hat{y}$ :

$$
\hat{y}:=\underset{y^{\prime} \in \mathcal{Y}(x)}{\arg \max }\left(\mathbf{w} \cdot \boldsymbol{\Phi}\left(x, y^{\prime}\right)+\Delta\left(y, y^{\prime}\right)\right)
$$

Previous work by MXfold gets $\hat{y}$ with loss-augmented decoding through exact search, but since the exact search-based inference requires $O\left(n^{3}\right)$ runtime, the inference is slow for long sequences, leading to a costly training process. Alternatively, we use LinearFold as a linear-time inference engine to get $y^{*}$, an approximation of $\hat{y}$ :

$$
y^{*}:=\underset{y^{\prime} \in \mathcal{Y}^{*}(x)}{\arg \max }\left(\mathbf{w} \cdot \boldsymbol{\Phi}\left(x, y^{\prime}\right)+\Delta\left(y, y^{\prime}\right)\right)
$$

where $\mathcal{Y}^{*}(x)$ is the search space of LinearFold and $\mathcal{Y}^{*}(x) \subseteq \mathcal{Y}(x)$.

\subsection{Convergence Analysis for sSVM with Inexact Search}

Though the training process can be accelerated with linear-time inexact search-based inference, the search error may lead to invalid updates and result in non-convergence. Next, we will show why sSVM's convergence property does not hold when applying inexact search.

First, we review the condition of exact search and analyze its convergence. Algorithm 1 presents the pseudocode of naive structured SVM training via Stochastic Gradient Descent (SGD), using exact search for loss augmented inference (line 6). We can prove that for a separable dataset sSVM training will make finite number of updates (before convergence) when doing exact search for inference.

Definition 1. D is structured $S V M$ separable with a margin of $\Delta\left(y, y^{\prime}\right)$, if there exists an oracle feature weight vector $\mathbf{u}$ with $\|\mathbf{u}\|=1$, s.t. it can correctly classify all examples in $\mathcal{D}$ with a gap of at least $\Delta\left(y, y^{\prime}\right)$. maximal margin $\delta(\mathcal{D})$ is the maximal such margin over all unit oracle vectors:

$$
\delta(\mathcal{D}):=\max _{\|\mathbf{u}\|=1} \min _{(x, y) \in \mathcal{D}} \min _{y^{\prime} \in \mathcal{Y}(x)-\{y\}} \mathbf{u} \cdot \Delta \boldsymbol{\Phi}\left(x, y, y^{\prime}\right)
$$

It is clear that $\forall(x, y) \in \mathcal{D}, \forall y^{\prime} \in \mathcal{Y}(x)-\{y\}, \delta(\mathcal{D}) \geq \Delta\left(y, y^{\prime}\right)$.

Definition 2. The diameter $R(\mathcal{D})$ of $\mathcal{D}$ is:

$$
R(\mathcal{D})=\max _{(x, y) \in \mathcal{D}} \max _{y^{\prime} \in \mathcal{Y}(x)-\{y\}}\left\|\Delta \mathbf{\Phi}\left(x, y, y^{\prime}\right)\right\|
$$


Theorem 1. For a structured $S V M$ separable dataset $\mathcal{D}$ with margin $\Delta\left(y, y^{\prime}\right)$, the stochastic gradient descent (SGD) algorithm (see Algorithm 1) will make finite number of updates (before convergence).

Proof. Training structured SVM via SGD is to minimize:

$$
\min _{\mathbf{w}} \frac{1}{2} \mathbf{w}^{T} \mathbf{w}-C(\mathbf{w} \cdot \Delta \mathbf{\Phi}(x, y, \hat{y})-\Delta(y, \hat{y}))
$$

The subgradient of the objective function is:

$$
\mathbf{w}-C \Delta \mathbf{\Phi}(x, y, \hat{y})
$$

Denote $\mathbf{w}^{\mathbf{k}}$ to be the weight vector at step $k$, and $\gamma$ to be the learning rate. At each step, $\mathbf{w}$ is updated as:

$$
\mathbf{w}^{\mathbf{k}+\mathbf{1}}=\mathbf{w}^{\mathbf{k}}-\gamma\left(\mathbf{w}^{\mathbf{k}}-C \Delta \mathbf{\Phi}(x, y, \hat{y})\right)
$$

We bound $\left\|\mathbf{w}^{\mathbf{k}+\mathbf{1}}\right\|$ in two directions. For detailed proof please refer to supporting information.

1. Upper bound:

$$
\left\|\mathbf{w}^{\mathbf{k}+\mathbf{1}}\right\|^{2}-\left\|\mathbf{w}^{\mathbf{k}}\right\|^{2}=-\gamma(2-\gamma)\left\|\mathbf{w}^{\mathbf{k}}\right\|^{2}+(\gamma C \Delta \boldsymbol{\Phi}(x, y, \hat{y}))^{2}+2(1-\gamma) \gamma C \mathbf{w}^{k} \cdot \Delta \boldsymbol{\Phi}(x, y, \hat{y})
$$

Since the update is because of a violation, by Equation 1 we have $(C>0)$ :

$$
\left\|\mathbf{w}^{\mathbf{k}+\mathbf{1}}\right\|^{2}-\left\|\mathbf{w}^{\mathbf{k}}\right\|^{2}<-\gamma(2-\gamma)\left\|\mathbf{w}^{\mathbf{k}}\right\|^{2}+(\gamma C \Delta \mathbf{\Phi}(x, y, \hat{y}))^{2}+2(1-\gamma) \gamma C \Delta(y, \hat{y})
$$

If we choose $0<\gamma<1$, by induction and Definition 1, 2, we can get the upper bound of:

$$
\left\|\mathbf{w}^{\mathbf{k}+\mathbf{1}}\right\|^{2}<k \gamma C\left(\gamma C R^{2}(\mathcal{D})+2(1-\gamma) \delta(\mathcal{D})\right)
$$

2. Lower bound:

$$
\mathbf{u} \cdot \mathbf{w}^{\mathbf{k}+\mathbf{1}}-\mathbf{u} \cdot \mathbf{w}^{\mathbf{k}}=-\gamma \mathbf{u} \cdot \mathbf{w}^{\mathbf{k}}+\gamma C \mathbf{u} \cdot \Delta \mathbf{\Phi}(x, y, \hat{y})
$$

By Definition 1 and upper bound of $\left\|\mathbf{w}^{\mathbf{k}}\right\|$, we get the lower bound as:

$$
\left\|\mathbf{w}^{\mathbf{k}+\mathbf{1}}\right\|^{2}>\left(-\gamma \alpha k^{\frac{3}{2}}+k \gamma C \delta(\mathcal{D})\right)^{2}
$$

where $\alpha=\sqrt{\gamma C\left(\gamma C R^{2}(\mathcal{D})+2(1-\gamma) \delta(\mathcal{D})\right)}$.

3. Combine upper and lower bound together, we have:

$$
k<\frac{(\gamma C \delta(\mathcal{D})+\alpha)^{2}}{(\gamma \alpha)^{2}}
$$

The key step in upper bound part is that the update from $\mathbf{w}^{\mathbf{k}}$ to $\mathbf{w}^{\mathbf{k}+\mathbf{1}}$ is because of a violation. When the inference uses exact search, the most violated structure $\hat{y}$ is guaranteed to score higher than ground truth structure $y$. However, when the search is inexact, for example beam search in LinearFold, it is possible that ground truth structure is not in the search space (i.e., $y \notin \mathcal{Y}^{*}(x)$ ). Thus, even if the predicted structure $y^{*}$ is different from $y$, it can still score lower, and the update is therefore invalid. When we do an invalid update, we shift $\mathbf{w}$ towards a wrong direction and the convergence may not hold. 


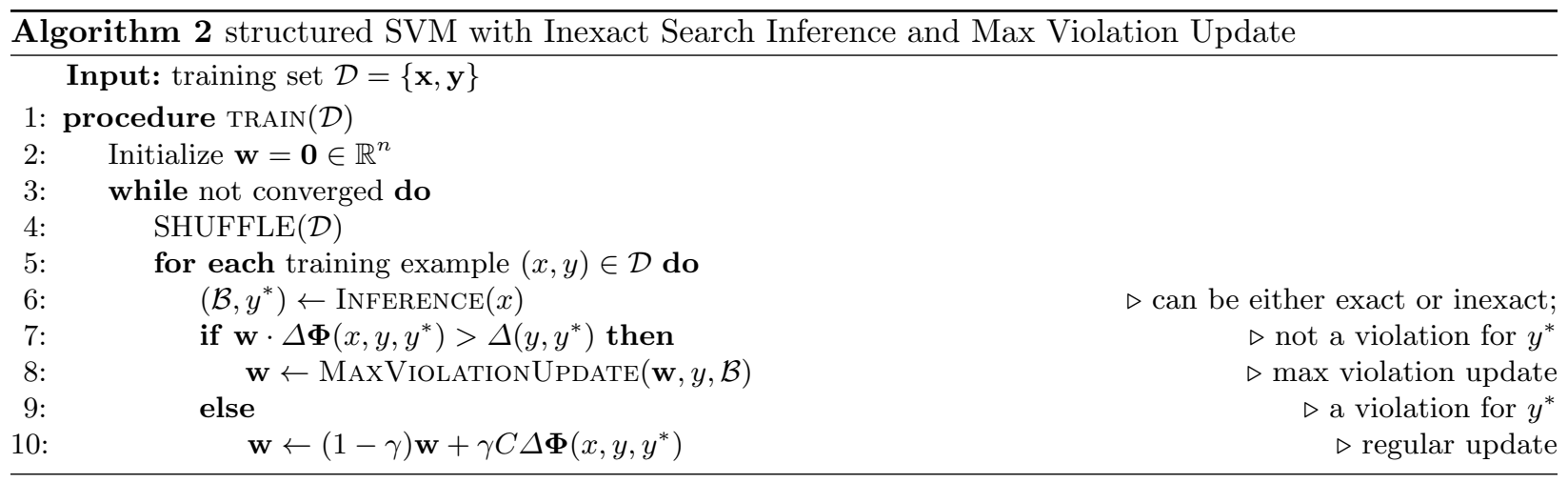

\subsection{Max Violation Update Strategy}

To remedy the non-convergence introduced by inexact search inference, We now propose the new framework of sSVM training, which uses max violation update strategy and can converge with inexact search.

The target of sSVM training is to learn a feature weight vector $\mathbf{w}$, which scores ground truth structure $y$ higher than any other structure, i.e., no violation for all examples. So, the main observation is each update is due to a violation. For exact search inference, if the predicted structure $\hat{y} \neq y$, it must be a violation and $\hat{y}$ must be the most violated structure. However, for inexact search inference, even if the predicted structure $y^{*} \neq y$, it is not necessary a violation since it is possible for $y$ to have a higher score than $y^{*}$.

With this observation, it is clear that the key solution is to make sure each update is for a violation, no matter if the search is exact. We introduce a max violation update strategy, which is a successful strategy in structured perceptron training. Basically, this strategy uses the most violated prefix of the ground truth structure and predicted structure for update. More formally, denote $y_{\mathrm{m} v}$ and $y_{\mathrm{m} v}^{*}$ as the max violation prefix of $y$ and $y^{*}$, and define them as:

$$
\left(y_{\mathrm{m} v}, y_{\mathrm{m} v}^{*}\right)=\underset{1 \leq j \leq n}{\arg \max } \mathbf{w} \cdot \Delta \mathbf{\Phi}\left(x, y_{[1: j]}, y_{[1: j]}^{*}\right)
$$

where $n$ is the sequence length and $j$ is each position in the sequence. $y_{[1: j]}$ is the prefix of $y$ up to $j$. Note that $y_{[1: j]}^{*}$ denotes the predicted substructure with the highest score up to $j$ in the searching space, and $y_{[1: j]}^{*} \in \mathcal{B}$, where $\mathcal{B}$ is the set of all such predicted substructures for every position $j$.

Algorithm 2 presents the pseudocode of sSVM training with max violation updated strategy. Now in line 6 it does not require an exact search (i.e., arg max) for inference, for example, we can use LinearFold as the inference engine. Then we check if $y^{*}$ results in a violation. If not, we do max violation update. Otherwise, we do regular update as in Algorithm 1.

\section{Results}

\subsection{Training Time}

First, we test the training time of our system, and compare with two machine learning-based systems, CONTRAfold and MXfold. We use ArchiveII dataset [16/18, 1] which contains 3,857 RNA sequences from 10 different RNA families. The average and max length of the sequences are 208.6 and 2,968. The 10 families (listed from the shortest to the longest) are tRNA, 5S rRNA, SRP, RNaseP, tmRNA, Group I Intron, Group II Intron, telomerase RNA, 16S rRNA and 23S rRNA.

Figure 1A compares per iteration runtime result among all three systems. We can see that CONTRAfold is the slowest and takes about 290 minutes, while MXfold is faster and takes about 15.37 minutes. Our system is the fastest and takes only 7.06 minutes, which is $41 \times$ faster than CONTRAfold training and $2.2 \times$ faster than MXfold training.

\footnotetext{
1 http://rna.urmc.rochester.edu/pub/archiveII.tar.gz
} 
A

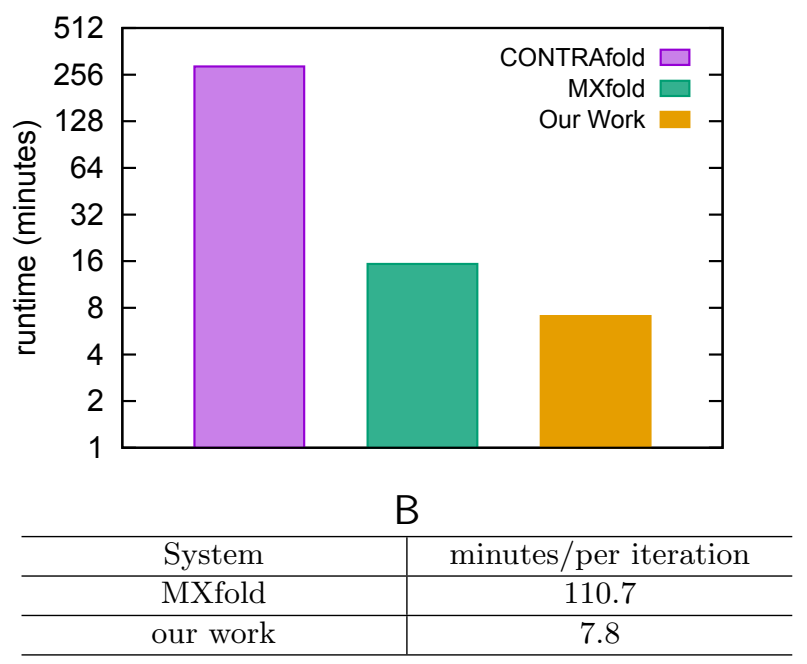

C

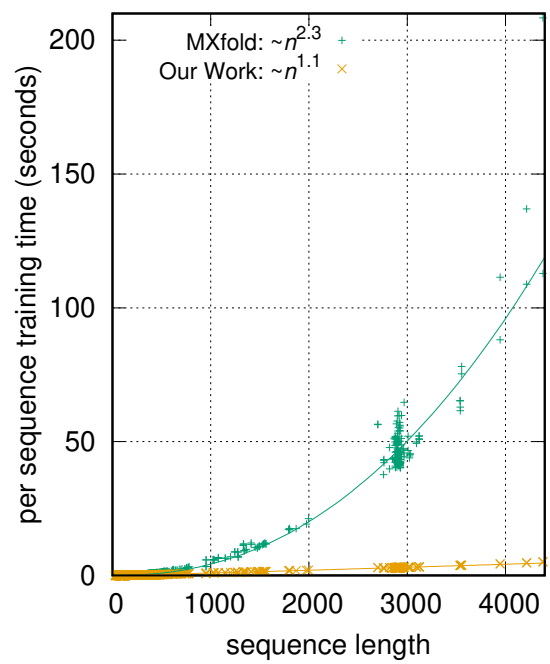

Fig. 1: Training runtime comparison between our system and the baselines. A: per iteration training runtime comparison between CONTRAfold, MXfold and our system on ArchiveII dataset. Note that the $y$-axis is in $\log$ scale. B: per iteration training runtime comparison between MXfold and our system on 23S rRNA dataset from bpRNA. C: per sequence training runtime comparison between MXfold and our system on these two datasets.

The speedup made by MXfold is mainly because sSVM training algorithm is faster than CRF used by CONTRAfold. Since MXfold still uses cubic algorithm for inference, we infer that if the dataset contains more long sequences, MXfold training will be much slower. To verify this, we collect long sequences in $23 \mathrm{~S}$ rRNA family from bpRNA dataset [5] $]^{2}$ and get a dataset containing 163 sequences, with average length 2713.5nt. Figure 1 $1 \mathrm{~B}$ shows the per iteration runtime comparison between MXfold and our system on this longer dataset. Since CONTRAfold is too slow, we do not include it. We can see that our system is about $14 \times$ faster, which comfirms that our system is much faster for training on longer sequences.

In addition, we test the runtime for each sequence to verify the linearity of our system. Figure $1 \mathrm{C}$ presents such comparison between MXfold and our system. We can see that our system scales almost linearly with the sequence length, while MXfold has super-quadratic runtime. The minor deviations from the theoretical runtimes are due to the majority of short sequences in ArchiveII dataset. For the longest sequence with length about 4381nt, MXfold takes about 208 seconds while our work only takes about 5 seconds, which is about $41 \times$ faster. We also notice that the runtime deviations of sequences with similar length for MXfold are much larger than our system, which suggests that our system can finish training within an accurate estimated time.

\section{$3.2 \quad$ Accuracy}

Next, we compare accuracy with both thermodynamics-based system and machine learning-based systems. We use cross-validation (leave-one-out) training on ArchiveII dataset, i.e., train on 9 families and test on the other family, to verify the learned models have strong generalization ability. We report Positive Predictive Value (PPV, the fraction of predicted pairs in the known structure) and sensitivity (the fraction of known pairs predicted $)$ for each family, and the overall PPV, sensitivity and F1-score $\left(\frac{2 \times P P V \times \text { sensitivity }}{\text { PPV+sensitivity }}\right)$ which are averaged over families. Following previous slipping method, we allow base pair to slip by one nucleotide [18. MXfold extends CONTRAfold features, and we also add these extra features into LinearFold when using it as the inference engine.

Figure 2 shows the per family and overall accuracies. We use 5 systems as baselines, the off-the-shelf versions of CONTRAfold, MXfold, LinearFold-C (LinearFold with machine learning-based model) and LinearFold-

2 http://bprna.cgrb.oregonstate.edu/ 
A

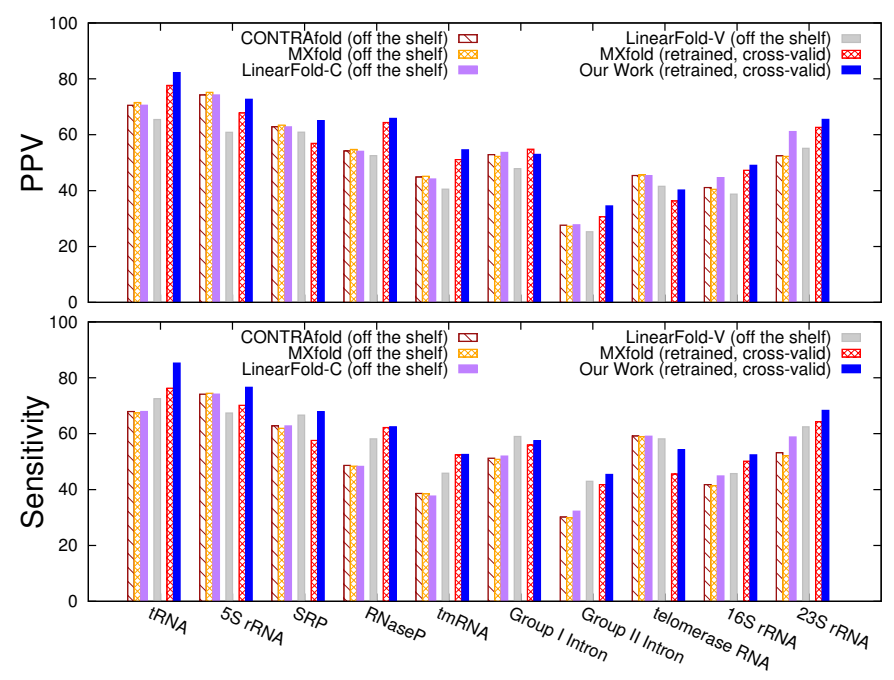

B

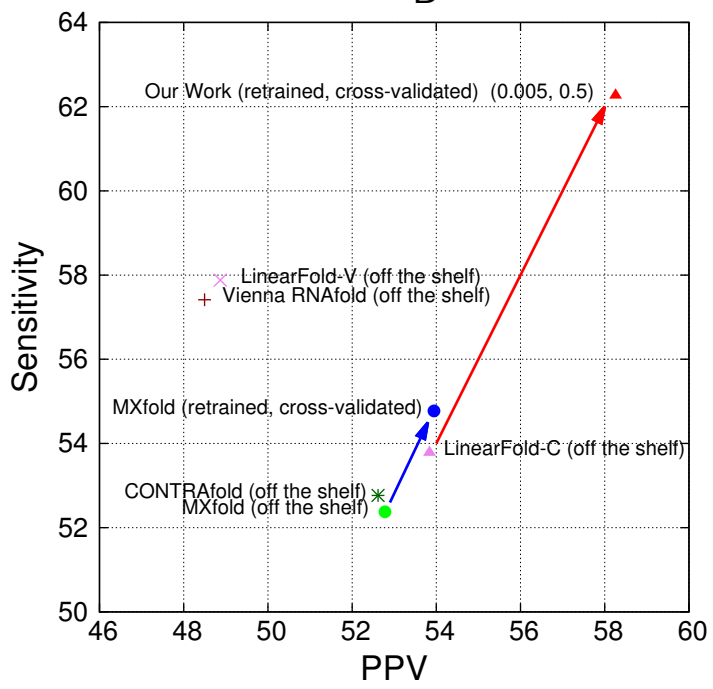

C

\begin{tabular}{c|c|c|c|c}
\hline Type & System & PPV & Sensitivity & F1-score \\
\hline \multirow{3}{*}{ off-the-shelf } & CONTRAfold & 52.62 & 52.77 & 52.69 \\
& MXfold & 52.78 & 52.38 & 52.58 \\
& LinearFold-C & 53.84 & 53.78 & 53.81 \\
& LinearFold-V & 48.87 & 57.88 & 53.00 \\
\hline \multirow{2}{*}{ cross-validation } & MXfold & 54.95 & 57.63 & 56.26 \\
& our work & $\mathbf{5 8 . 4 6}$ & $\mathbf{6 2 . 3 3}$ & $\mathbf{6 0 . 3 3}$ \\
\hline
\end{tabular}

Fig. 2: Accuracy comparison between different systems. A: per family PPV and sensitivity comparison. B: overall PPV-sensitivity-tradeoff comparison. $(0.005,0.5)$ are the values of hyper-parameters $(\alpha, \beta)$. C: table for overall PPV, sensitivity and F1-score, averaging over all families.

V (LinearFold with thermodynamics-based model), as well as retrained MXfold. CONTRAfold is too slow for retraining, so we do not include retrained CONTRAfold as a baseline. We do cross-validation training for our system and MXfold for 100 epochs.

Figure 2 $2 \mathrm{~A}$ shows that our system has the highest PPV on all families except 5S rRNA, Group I Intron and telomerase RNA, as well as the highest sensitivity on all families except Group I Intron and telomerase RNA. Note that off-the-shelf machine learning-based systems may produce better results as they may have corresponding families in their training sets. Our system outperforms retrained MXfold on both PPV and sensitivity for all families, except for PPV of Group I Intron. The results verify that our linear-time training system can learn better models, and also shows that our system has a good generalization ability of learning models which can be used across families.

We demonstrate PPV-sensitivity-tradeoff in figure $2 \mathrm{~B}$, and show our work's PPV-sensitivity with selected hyper-parameters $(\alpha=0.005, \beta=0.5)$. We can see that our system achieves the highest overall PPV and sensitivity among all systems, including machine learning-based systems and thermodynamics-base systems, by big margins. Figure $2 \mathrm{C}$ gives the exact number of overall PPV, Sensitivity and F1-Score in different systems. Our system improves off-the-shelf LinearFold-C by $+4.62 \%$ for PPV,$+8.55 \%$ for sensitivity and $+6.51 \%$ for F1-score, and outperforms retrained MXfold by $+3.51 \%,+4.70 \%,+4.07 \%$ on PPV, sensitivity and F1-Score, separately. Compared with some other off-the-shelf systems, the improvement is even bigger. For example, Our system outperforms the off-the-shelf CONTRAfold by $+5.84 \%,+9.56 \%,+7.64 \%$ on PPV, sensitivity and F1-Score, separately.

We borrow MXfold feature template for all experiments in figure2 but we notice that even with the basic feature template in CONTRAfold (about 336 non-zero features), our system achieves a similar accuracy as using MXfold feature template (around 4,000 non-zero features). Table 2 shows the accuracy comparison for systems with different feature templates. We can see that PPV and sensitivity changes of our system 
Rezaur Chowdhury et al.

\begin{tabular}{c|c|c|c|c}
\hline Features & System & PPV & Sensitivity & F1-score \\
\hline \multirow{2}{*}{ basic } & CONTRAfold (off-the-shelf) & 52.62 & 52.77 & 52.69 \\
& our work (retrained) & $\mathbf{5 8 . 3 5}$ & $\mathbf{6 2 . 3 2}$ & $\mathbf{6 0 . 2 7}$ \\
\hline \multirow{3}{*}{ rich } & MXfold (retrained, w/o thermodynamic model) & 54.42 & 54.82 & 54.62 \\
& MXfold (retrained, w/ thermodynamic model) & 54.95 & 57.63 & 56.26 \\
& our work (retrained) & $\mathbf{5 8 . 4 6}$ & $\mathbf{6 2 . 3 3}$ & $\mathbf{6 0 . 3 3}$ \\
\hline
\end{tabular}

Table 2: Accuracy comparison of systems with different feature templates.

\begin{tabular}{c|c|c|c|c}
\hline Beam Size & Update Strategy & PPV & Sensitivity & F1-score \\
\hline \multirow{2}{*}{1} & regular & 14.94 & 12.25 & 13.46 \\
& max violation & 17.38 & 13.62 & 15.27 \\
\hline \multirow{2}{*}{5} & regular & 29.93 & 29.39 & 29.66 \\
& max violation & 33.02 & 31.80 & 32.39 \\
\hline \multirow{2}{*}{50} & regular & 55.80 & 59.64 & 57.66 \\
& max violation & 56.87 & 60.11 & 58.44 \\
\hline \multirow{2}{*}{100} & regular & 57.12 & 61.73 & 59.34 \\
& max violation & 58.16 & 61.39 & 59.73 \\
\hline
\end{tabular}

Table 3: Comparison between regular and max violation update strategy for different beam sizes on ArchiveII cross validation training (run for 10 epochs).

with different feature template are very small, only $0.11 \%$ and $0.01 \%$, separately. In addition, even with a much smaller feature template, our system is better than MXfold. On the other hand, MXfold integrates thermodynamic model in its system, which helps for generalization and makes it a mixture of learning-based and thermodynamics-base system. If thermodynamic model is disabled and train for a pure learning model for MXfold, the PPV and sensitivity drop $-0.53 \%$ and $-2.81 \%$, separately. Compared to this pure learning-based MXfold, our system's accuracy improvement is even more salient.

\subsection{Update Strategy Impact}

In section 2.3 we introduce max violation update strategy as a fix for invalid update in sSVM training with inexact search inference. Here we investigate the impact of different update strategies, i.e., regular update and max violation update, on accuracy.

Table 3 shows the accuracy changes between regular and max violation update strategies on ArchiveII cross validation training. Since the search quality is highly related to beam size, we show the comparison between different beam sizes too. We can see that with small beam sizes $b=1$ and $b=5$, the search quality is bad, thus max violation update strategy leads to a better accuracy. With beam size increasing to $b=50$ or even $b=100$, the search quality for most short sequences is good (i.e., doing exact search for families such as tRNA and 5S rRNA), and invalid update becomes fewer, resulting in closer accuracies between two different update strategies. This confirms invalid updates are the key for sSVM training with inexact search.

Since ArchiveII dataset is very diverse, which contains both very long (about 2,900 nt) and very short (about $50 \mathrm{nt}$ ) sequences, it is hard to choose a balanced beam size for training, i.e., a small beam size is suitable for short families but bad for long families, and a large beam size makes the search for most sequences become exact search. However, on some other less diverse dataset, for example S-Processed dataset (a data set used by CONTRAfold v2.02 training, originally from S-full dataset [2] $]^{3}$, the impact is more salient.

Figure 3 shows the impact of different update strategy on S-Processed dataset training. Figure $3 \mathrm{~A}$ shows that for beam size $b=5$, max violation update strategy leads to a better accuracy. Compared to regular update, max violation update helps to gain about $+5 \%$ increase on F1-score. In addition, the curve of regular update drops after 500 seconds, but max violation update curve keeps increasing in all the training process. Figure $3 \mathrm{~B}$ visualizes an example from S-Processed test set. This example's length is 106nt, and is part of

\footnotetext{
3 http://www.rnasoft.ca/CG/
} 
A

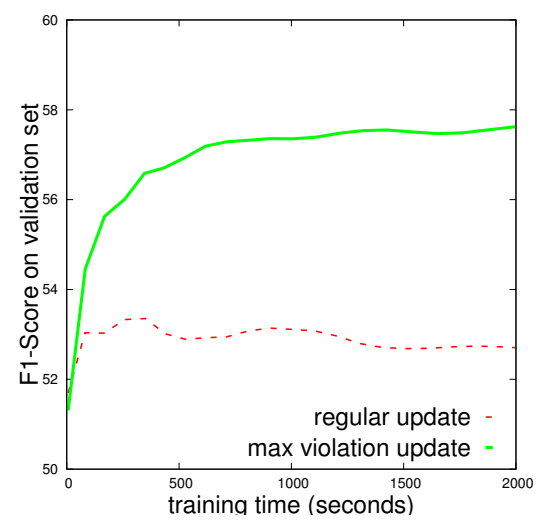

B

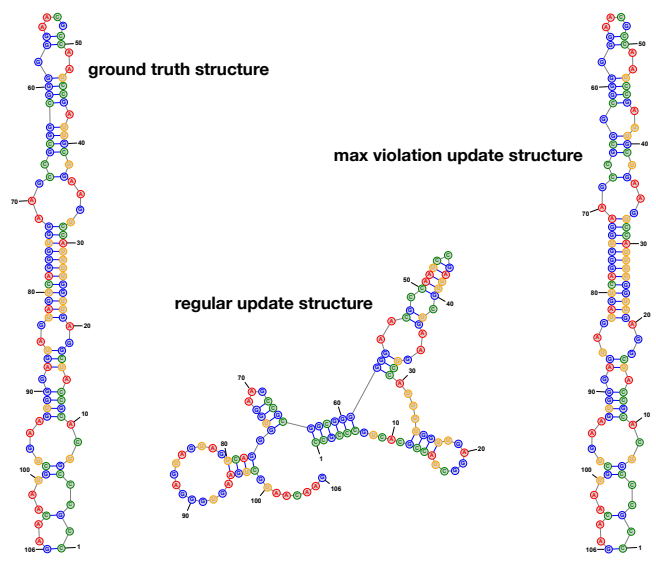

Fig. 3: Impact of different update strategy on S-Processed dataset training. A: F1-score curves of training processes with two different update strategies for beam size 5. B: An example showing that max violation update strategy gives a more accurate structure prediction. RNA secondary structures are drawn with StructureEditor.

a 16S rRNA sequence. We show three structures of this example, ground truth structure, regular update structure and max violation update structure. We can see that prediction structure with max violation update strategy is almost the same as the ground truth structure, and its PPV and sensitivity are 96.97\% and $94.12 \%$, separately. Not as good as max violation update structure, prediction structure with regular update strategy is very different from the ground truth structure, which incorrectly predicts all of the base pairs.

\section{Discussion}

Machine learning-based methods for RNA secondary structure prediction can learn feature weights from known structures, and address the inaccurate measurement of thermodynamic parameters due to experimental limitation. However, training with big datasets containing diverse sequences, which is beneficial for learning-based methods, is either impossible or very expensive due to the slowness of current learning systems. To address this issue, we propose a new machine learning-based system, which can learn to fold in linear time. Our system uses the successful linear-time prediction engine LinearFold as inference, and uses structured SVM, a much faster machine learning algorithm than CRF used by CONTRAfold, for training procedure. Furthermore, we utilize a max violation update strategy to address the non-convergence issue introduced by using LinearFold as inexact search inference, and generalize the naive sSVM algorithm to a max violation update version of sSVM.

We confirm that:

1. The training speed of our system is $41 \times$ faster than CONTRAfold for one epoch even on a diverse dataset (average length $208.6 \mathrm{nt}$ ). On a dataset with more long sequences (average length $2713.5 \mathrm{nt}$ ), our system is $14 \times$ faster than MXfold. The training time for each sequence increases linearly with sequence length. See figure 1 .

2. The accuracy of our system outperforms both thermodynamics-based systems and machine learningbased systems, and achieves the highest accuracy in most families. Our system has a strong generalization ability, which can learn good models from cross-validation training. See figure 2.

3. Max violation update strategy leads to accuracy improvement. See figure 3

Our system can be extended to deep learning-based RNA folding system. Now our model still uses manually designed features, but it can also be built on some well-designed neural networks, which can 
automatically extract implicit features. With these features, it is possible to get a more accurate RNA folding prediction system.

\section{References}

1. Akiyama, M., Sato, K., Sakakibara, Y.: A max-margin training of RNA secondary structure prediction integrated with the thermodynamic model. bioRxiv (2017)

2. Andronescu, M.: Computational approaches for RNA energy parameter estimation. PhD thesis (2008)

3. Collins, M.: Discriminative training methods for hidden Markov models: Theory and experiments with perceptron algorithms. In: Proceedings of the 2002 Conference on Empirical Methods in Natural Language Processing (EMNLP 2002). pp. 1-8. Association for Computational Linguistics (Jul 2002). https://doi.org/10.3115/1118693.1118694, https://www.aclweb.org/anthology/W02-1001

4. Collins, M., Roark, B.: Incremental parsing with the perceptron algorithm. In: Proceedings of the 42nd Annual Meeting of the Association for Computational Linguistics (ACL-04). pp. 111-118. Barcelona, Spain (Jul 2004). https://doi.org/10.3115/1218955.1218970, https://www.aclweb.org/anthology/P04-1015

5. Danaee, P., Rouches, M., Wiley, M., Deng, D., Huang, L., Hendrix, D.: bpRNA: large-scale automated annotation and analysis of RNA secondary structure. Nucleic Acids Research 46(11), 5381-5394 (2018)

6. Do, C., Woods, D., Batzoglou, S.: CONTRAfold: RNA secondary structure prediction without physics-based models. Bioinformatics 22(14), e90-e98 (2006)

7. Durbin, R., Eddy, S.R., Krogh, A., Mitchison, G.J.: Biological sequence analysis: Probabilistic models of proteins and nucleic acids (1998)

8. Eddy., S.R.: Non-coding RNA genes and the modern rna world. Nature Reviews Genetics 2(12), 919-929 (2001)

9. Huang, L., Fayong, S., Guo, Y.: Structured perceptron with inexact search. In: Proceedings of the 2012 Conference of the North American Chapter of the Association for Computational Linguistics: Human Language Technologies. pp. 142-151. Association for Computational Linguistics, Montréal, Canada (Jun 2012), https://www.aclweb.org/ anthology/N12-1015

10. Huang, L., Zhang, H., Deng, D., Zhao, K., Liu, K., Hendrix, D.A., Mathews, D.H.: LinearFold: linear-time approximate RNA folding by 5'-to-3' dynamic programming and beam search. Bioinformatics 35(14), i295-i304 (07 2019). https://doi.org/10.1093/bioinformatics/btz375, https://doi.org/10.1093/bioinformatics/btz375

11. Knudsen, B., Hein, J.: RNA secondary structure prediction using stochastic context-free grammars and evolutionary history. Bioinformatics 15(6), 446-454 (06 1999). https://doi.org/10.1093/bioinformatics/15.6.446, https://doi.org/10.1093/bioinformatics/15.6.446

12. Knudsen, B., Hein, J.: Pfold: RNA secondary structure prediction using stochastic context-free grammars. Nucleic Acids Research 31(13), 3423-3428 (2003)

13. Kung, J.T.Y., Colognori, D., Lee, J.T.: Long noncoding RNAs: Past, present, and future. Genetics 193(3), 651-669 (2013)

14. Lafferty, J., McCallum, A., Pereira, F.C.: Conditional random fields: Probabilistic models for segmenting and labeling sequence data. In: Proceedings of the 18th International Conference on Machine Learning 2001 (ICML 2001). pp. 282-289 (2001)

15. Lorenz, R., Bernhart, S.H., Zu Siederdissen, C.H., Tafer, H., Flamm, C., Stadler, P.F., Hofacker, I.L.: ViennaRNA package 2.0. Algorithms for Molecular Biology 6(1), 1 (2011)

16. Mathews, D.H., Sabina, J., Zuker, M., Turner, D.H.: Expanded sequence dependence of thermodynamic parameters improves prediction of RNA secondary structure. Journal of molecular biology 288(5), 911-940 (1999)

17. Mathews, D.H., Turner, D.H.: Prediction of RNA secondary structure by free energy minimization. Current Opinion in Structural Biology 16(3), 270-278 (2006)

18. Sloma, M., Mathews, D.: Exact calculation of loop formation probability identifies folding motifs in RNA secondary structures. RNA, 22, 1808-1818 (2016)

19. Tsochantaridis, I., Hofmann, T., Joachims, T., Altun, Y.: Support vector machine learning for interdependent and structured output spaces. In: ICML (2004) 